\title{
Einige Bemerkungen zu lautlichen und morphologischen Gemeinsamkeiten und Unterschieden zwischen den Dorfmundarten von Lichwe und Riebnig im Schönhengstgau
}

\author{
Richard Rothenhagen
}

0. Im Rahmen eines staatlichen und internationalen Projekts beschäftigen wir uns seit 2001 mit den ehemaligen deutschen Mundarten (im ff. MA) in Mähren und Österreichisch Schlesien. Wir fahren ins Terrain, erstellen Aufnahmen und arbeiten mit Fragebüchern, um Vergleiche anstellen zu können.

Die bisher zur Verfügung stehende Fachliteratur zu den MA auf diesem Gebiet stammt ausschließlich aus der Zeit vor dem Zweiten Weltkrieg, s. z. B. MATZKE (1922) oder BENESCH (1938), wobei das bearbeitete Wortmaterial oft noch aus der Zeit vor dem Ersten Weltkrieg stammt. Jedoch auch eine MA ändert sich im Laufe der Zeit, und wenn wir mit unseren Gewährspersonen arbeiten, dann spiegeln ihre Äußerungen eher den Sprachzustand vor und während des Zweiten Weltkrieges wider.

Eben auf Grund der erwähnten Literatur wurde bei der Einteilung der Schönhengster MA Lichwe (Libchavy) zu einem besonderen MA-Gebiet gerechnet, während Riebnig (Rybník), zwar mit einigen Besonderheiten (MATZKE: 1922, 226), in die sog. Landskroner MA einbezogen wurde. Unsere Untersuchungen belegen aber, dass diese Einteilung in Zweifel gezogen werden kann, wie einige Vergleiche zu den MA von Rudelsdorf (Rudoltice) und Landskron (Lanškroun) zeigen werden.

1. Betrachten wir nun erst einmal einige lautliche Erscheinungen dieser MA.

1.1. Alle die genannten MA gehören zu den sog. Apokope-MA, d. h. auslautendes [e] fällt weg, wie z. B. in bi Azd (Bürste) z dobl (Stange) and (Ente) g He (Kette) Ve $5 b$ (Wespe).

1.2. Die MA von Lichwe und von Riebnig vokalisieren das [r] zu [i], wenn es zwischen einem [a] und einem Velaren oder einer Konsonantengruppe mit Velarem an erster Stelle zu stehen kommt; so heißt es also in Lichwe z. B. g Hwobg (Quark) moli g (Markt) und in Riebnigg HV obg (Quark) z do 5 g (stark). In Lichwe bezieht sich diese Vokalisierungsregel sogar auch auf das [u], z. B. vui $\$ d$ (Furche). Diese Erscheinung ist sowohl in der Rudelsdorfer als auch in der Landskroner MA nicht zu beobachten.

1.3. Beide MA haben im Anlaut den Affrikaten [pf], z. B. bv l u (Pflug), b v I ugNK(pflücken) in Lichwe; bv I i gNK (pflücken), bvä r A (Pfarrer) in 
Riebnig, worauf auch MATZKE (1922, 62f.) hinweist. Im Inlaut und im Auslaut wird diese Regel in Riebnig auch eingehalten, denn man sagt e $\bar{b} \mathrm{v} \mid \mathrm{K}$ (Apfel) und do (Topf), während es in Lichwe o b | K (Apfel) und do (Topf) heißt, worauf MATZKE (1922, 80-85) ebenfalls hinweist. In Rudelsdorf verhält man sich in dieser Hinsicht wie in Riebnig, in Landskron ist man inkonsequent, denn am Ende tritt [bv] nicht auf, wie z. B. in doly.

1.4. Wenn es zu einem [n]-Ausfall kommt, stellt sich in Lichwe und Riebnig dafür oft eine Nasalierung ein. So sagt man z. B. in Riebnig: d s o \%o(Zahn), z i $\mathrm{a}=$ (Schienbein), s̈̈z (Sohn), di z di g (Dienstag). Obwohl in dieser MA diese Erscheinung ziemlich konsequent auftritt, kann es gelegentlich trotzdem dazu kommen, dass diese Nasalierung nicht eintritt, z. B. in hi ka (Hühnchen, ein Kücken, das eine Henne wird.) oder haka (Hähnchen, ein Kücken, das ein Hahn wird.) In Lichwe ist die Nasalierung weit weniger verbreitet, man sagt zwar $s$ ä (Sense), u aber auf der anderen Seite $z d a \dot{F} 5$ (Stein), gr i = (grün), z u 5 (Scheune). In Rudelsdorf ist die Nasalierung selten, und wenn sie dennoch erfolgt, dann sehr schwach, z. B. im Wort $\mathrm{s}$ ä (Sense); in Landskron ist sie ganz verschwunden, z. B. $z d a=$ (Stein), së as (Sense), z a i 5 (Scheune).

1.4.1. Interessant ist, dass bei der Präposition in in allen vier MA das [n] ebenfalls wegfällt; jedoch tritt hierbei in keiner von ihnen eine Nasalierung auf, überall heißt es $i=d A \quad z d u$ (in der Stube). MATZKE (1922) spricht in diesem Zusammenhang davon, dass (zu seiner Zeit) die Nasalierung im Schwinden begriffen war. Somit lässt sich annehmen, dass die MA von Riebnig in erster Linie noch die älteren Formen bewahrt hat.

1.5. Beide MA verwenden für das Possessivpronomen [unser] die Form undë . Man sagt also sowohl in Lichwe als auch in Riebnig z. B. nd ë ( g Ho lo (unsere Katze), während wir in Rudelsdorf und in Landskron die standardnäheren Formen uns $\mathrm{E}$ re 5 bzw. uns vorfinden.

1.6. Für in der Silbe oder im Wort anlautendes [w] erscheint in Lichwe konsequent ein [b], also z. B. büka (wer), büg (Weg) z bi верё̈ (Spinnennetz). In Riebnig dagegen erscheinen diese Wörter mit [w]: Va $V$ a g , z b i G E V e . Es ist jedoch anzunehmen, dass es diese Erscheinung auch in Riebnig gegeben hat, denn sie ist teilweise noch erhalten. So sagt man z. B. mi A büh I i a ha hil $\mathrm{Fa}$ (Wir werden lieber nach Hause gehen.) Anscheinend handelt es sich hier um eine Erscheinung, die allmählich verschwunden ist (zumindest was Riebnig betrifft) und nur noch reliktartig 
vorkommt. Damit wäre es in diesem Falle so, dass eher Lichwe den älteren Stand erhalten hat. Weder in Rudelsdorf noch in Landskron kommen wir mit dieser Erscheinung in Berührung.

1.7. In beiden MA gibt es den Diphthong [eu], der z. B. aus dem mhd. -iuund anderen Lautkombinationen entstanden ist, $d . h$. in beiden MA sagen wir z. B.: a 4 noi $G \mathrm{glOg}$ (eine neue Glocke), hobG (heuer), I ObBnK

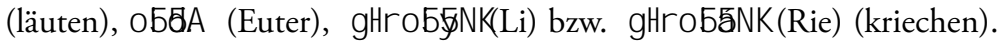
In Lichwe kann es aber passieren, dass an anderen Stellen, wo man ein [eu] erwartet würde, auf einmal ein [ui] erscheint. So sagt man z. B. s $\mathbf{\text { E }}$ । ui $\mathbf{Q}$. nK (sie läuten), nui \& (neuer), a u v r u i Đna (aufräumen), d. h. demnach, dass z. B. in den konjugierten Formen des Verbs, läuten' ein anderer Laut wie im Infinitiv erscheinen kann. In Riebnig ist man in dieser Frage „konsequenter". Nehmen wir z. B. das Verb v O 0 G $\mathrm{g}$ KK (fliegen). Hier ist es z. B. so, dass wir diesen Diphthong in allen Personen haben, z. B. i

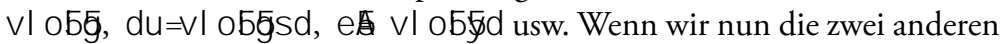
Orte betrachten, dann stellen wir fest, dass Rudelsdorfähnliche Verhältnisse zeigt wie Riebnig, denn man sagt dort z. B. auch $n O 55$ oder $05 \mathrm{BA}$, aber in Landskron erscheint für diesen Diphthong ein [ei], also n a 5 (neu), a i $\mathbf{B} \mathrm{A}$ (Euter), ha i 5 (Heu).

1.7.1. Eine ganz besondere Eigenart der Lichwer MA besteht darin, dass der [eu]-Diphthong am Wortende zu einem Triphthong werden, kann wie z. B. in holi 5 (Heu). Auf diese Besonderheit weist auch MATZKE $(1922,157)$ hin.

2. Kommen wir nun zu einigen grammatikalischen Erscheinungen. Hier werden wir feststellen müssen, dass die Gemeinsamkeiten relativ gering sind.

\subsection{Betrachten wir zunächst das Verb:}

2.1.1. Bereits in der Bildung der Tempora ergeben sich zwischen den einzelnen MA Abweichungen. So wird z. B. von den Verben „stehen“ und „liegen“ das Perfekt unterschiedlich gebildet. In Lichwe erscheint es mit dem Verb

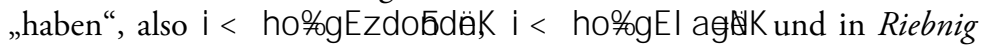
mit dem Verb „sein“: e is gEzdobduk bzw. e is gEl ag Die Bildung des Perfekts mit dem Verb „sein“ ist auch in Rudelsdorf und in Landskron zu beobachten. In Lichwe wird also die ostmitteldeutsche Form vorgezogen, während sich die anderen Orte an die oberdeutsche Art halten. 
2.1.2. Der Infinitiv kann endungslos sein. Das trifft auf die Verben zu, die im Standarddeutschen auf [-eln] enden, und zwar in beiden DorfMA. So stoßen wir z. B. auf folgende Wörter: (Li) z i me $b / z$ i ma $\approx \sim($ Rie) (schimmeln), (Li) hege $/$ he a A ba Lichwe können vereinzelt auch andere Verben ohne n-Endung erscheinen, jedoch handelt es sich hier um einen n-Wegfall, der nicht mit den oben genannten Regelmäßigkeiten in Verbindung gebracht werden sollte. Das betrifft z. B. die Verben ,hauen“ $-h a=$ und „sehen “- $s$ $\ddot{\theta}=$ In Riebnig bleibt bei diesen Verben das [n] erhalten: ha f, $\mathbf{s} \ddot{A}$. Diese Erscheinung ist weder in Rudelsdorf noch in Landskron zu beobachten.

2.1.3. Der Imperativ vom Verb sein lautet in beiden MA $b i \neq d$. h. dass bei der Bildung von der 2. Ps. Sg. ausgegangen wird, also z. B.: $b i=z d i b$ (Li), bi =r u $=\mathrm{g}$ (Rie), allerdings kann in Riebnig auch die Form sä i 5 auftreten. Diese letztere Form ist die einzige in Rudelsdorf und Landskron vertretene Form.

2.1.4. Das Konditional bzw. der Konjunktiv wird sowohl in Lichwe als auch in Riebnig mit Hilfe des Verbs "tun“ zum Ausdruck gebracht. So klingt der Satz: Wenn er endlich aufstünde! in beiden MA folgendermaßen: bë $\overline{\text { A }}$ ? e $\overline{a d l}$ i a uvzdi de d! oder der Nebensatz: Wenn er läge, täte ihm nichts weh: bë́A I i glKde\&, de i nigs Vi =In Rudelsdorf und Landskron wird statt $d e \notin$ eher eine Form mit me $3 d$ vorgezogen, z. B.: Véan I 0 äKme $5 d$,Wenn er ihn doch ließe.

\subsection{Wenden wir uns nun den Substantiven zu.}

2.2.1. Eine ähnliche Erscheinung wie bei den Verben auf -eln können wir auch bei den Substantiven beobachten. Substantive, die im Singular auf ein [1] enden, haben keine Pluralendung, sondern schieben vor das [1] einen Vokal ein. Das betrifft wiederum beide Dorf-MA, z. B.:

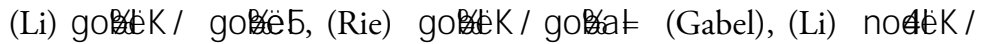

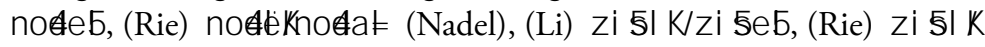
$z$ i $s$ a $F$ (Schüssel). In Lichwe kann dies auch Substantive betreffen, die als Diminutivformen im Singular ein [l] haben, wie z. B.: Vo 阽 K K/ V o 数e 5 (Wade), g Ho $\mathrm{I}$ K/g Ho G e b (Kanne).

2.2.2. In beiden MA können auch Pluralformen auftreten, die wir vom Standarddeutschen her nicht kennen. Dies betrifft vor allem 
Umlautformen: So lautet sowohl in Lichwe als auch in Riebnig der Plural von Hund hund - hi nd, eine Pluralform, die auch in den weiter nordöstlich vertretenen mährisch-schlesischen MA sehr häufig vorkommt. In Lichwe finden wir außerdem solche vom Standard abweichende Pluralformen wie: vai $\mathbb{d}$ o / vai $\mathbb{d}$ de (Feiertag/Feiertage), ma us I mui 5 (Maus/Mäuse), aber auch Formen, die uns bekannt sind: haud /hob (Haut/Häute). In Riebnig treten solche für den Standard ungewöhnlichen Plurale selten auf, interessant ist nur z. B. der Plural von „Koffer": g Huv e ( / g Hi f e / Die unter 2.2.1 und 2.2.2. angeführten Erscheinungen sind weder für Rudelsdorf noch für Landskron markant.

2.2.3. In beiden Dorf-MA und auch in den anderen zum Vergleich herangezogenen MA von Rudelsdorf und Landskron ist das Wort Bach ein Maskulinum, was auf stärkeren oberdeutschen Einfluss schließen lässt - das betrifft auch Rudelsdorf und Landskron -, weil in den ostmitteldeutschen MA dieses Substantiv sonst ein Femininum ist.

2.3. In Bezug auf die Zahlwörter wäre noch zu sagen, dass die Riebniger MA die einzige von den genannten MA ist, die bei der Zahl zwei noch unterschiedliche Formen für alle drei Geschlechter aufweist, denn in Riebnig heißt es: $d s$ wi =me be ( (zwei Männer), ds wu =g Hi fa 4 (zwei Kühe),

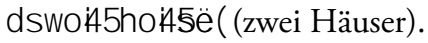

2.4. Bei den Adverbien und Pronomina wäre noch zu erwähnen, dass in beiden untersuchten Dorf-MA z. B. das Interrogativpronomen wer und einige lokalbezogene Adverbien durch ein auslautendes [a] verstärkt werden, also (Li) bü a $4 \mathrm{~V}$ a 4 (Rie), (Li/Rie) ha 4 (her), (Li/Rie) dë 4 a (dort) 4

3. Wenn wir nun noch einmal zum Ausgangspunkt zurückkehren, dann können wir sagen, dass die Gemeinsamkeiten zwischen Lichwe und Riebnig eher größer sind als die Gemeinsamkeiten zwischen Riebnig, Rudelsdorf und Landskron und damit von Riebnig zu dem restlichen Landskroner Gebiet. Die einzige große Gemeinsamkeit, die alle MA verbindet, ist die Apokope.

Die Gemeinsamkeiten zwischen Lichwe und Riebnig werden zwar durch kleinere Abweichungen etwas abgeschwächt, aber diese Abweichungen sind keinesfalls größer als diejenigen, die Lichwe und Riebnig von Rudelsdorf und Landskron trennen, eher das Gegenteil ist der Fall. Aus diesem Grunde ist es unserer Meinung nach auf keinen Fall berechtigt, Riebnig zum Gebiet der Landskroner MA zu rechnen und Lichwe nicht. 
Eine befriedigende Antwort auf die Frage, wie die MA zu gruppieren wären, kann wohl erst nach einigen weiteren Untersuchungen gegeben werden. Allerdings kann bereits jetzt gesagt werden, dass trotz aller aufgezeigter interner Differenzierung die Unterschiede zwischen den bisher genannten vier MA im Vergleich zu den Unterschieden zu den anderen im östlichen, mittleren und südwestlichen Schönhengst vertretenen MA minimal sind. Die Unterschiede zu diesen anderen MA sind so immens, dass es zu Verständnisschwierigkeiten kommen kann, was man von den vier behandelten MA trotz aller Unterschiede nicht behaupten kann. Die einzigen MA im Schönhengst, die außerdem noch starke Ähnlichkeiten mit den zuletzt genannten MA aufweisen, wären die MA von Brüsau (Březová) und den sich nach Süden anschließenden Industriedörfern, evtl. auch noch die MA von Kornitz (Chornice) und Dörfles (Víska).

Das von MATZKE (1922) für die Landskroner MA umrissene Gebiet reicht von der Nordgrenze (außer Schönwald /Strážná/) des Schönhengstes nach Süden bis etwa Mährisch-Trübau (Moravská Třebová), im Osten bis etwa Budigsdorf(Krasíkov) und im Westen an die tschechische Sprachgrenze. Im Falle von Riebnig müsste man sich entscheiden: Entweder man zählt beide Dörfer, d. h. sowohl Lichwe als auch Riebnig zu diesem MA-Gebiet oder man lässt sie als selbstständige MA gelten. Die Entscheidung sollte erst nach Abschluss aller Untersuchungen fallen, die für den Schönhengst auf Grund unseres Materials noch anstehen.

\section{Zusammenfassung:}

In diesem Beitrag werden die Restbestände der deutschen Mundarten in Mähren und Österreichisch Schlesien untersucht. Am Beispiel der Mundart von Lichwe (Libchavy) und Riebnig (Rybník) werden einige, vornehmlich lautliche und grammatikalische Erscheinungen dieser Mundarten betrachtet. Bei der Einteilung der Schönhengster Mundarten wurde Lichwe zu einem besonderen Mundarten-Gebiet gerechnet, während Riebnig der sog. Landskroner Mundart zugeordnet wurde. Die in diesem Beitrag präsentierten Untersuchungen belegen, dass diese Einteilung in Zweifel gezogen werden kann.

Schlüsselwörter:

Mundarten in Mähren - Mundarten in Österreichisch Schlesien - mährische Mundarten Lichwe - Riebnig - Schönhengster Mundart - Landskroner Mundart - Schönhengst Landskron 


\section{Literatur}

BAUFELD (1996): Baufeld, Christa. Kleines frühneuhochdeutsches Wörterbuch. Tübingen: Max Niemeyer, 1996.

BENESCH (1938): Benesch, Irmfried. Lautgeographie der Schönhengster Mundarten. Brünn, Prag, Leipzig, Wien: Rudolf M. Rohrer, 1938.

BERANEK (1936): Beranek, Franz. Beiträge zur Kenntnis sudetendeutscher Mundarten. Die Mundart von Südmähren. Reichenberg: Verlag der Anstalt für sudetendeutsche Heimatforschung, 1936.

FRINGS (1922): Frings, Theodor. Sprache und Siedlung im mitteldeutschen Osten. Leipzig: Verlag von S. Hirzel, 1922.

HENNINGS (2003): Hennings, Thordis. Einführung in das Mittelhochdeutsche. Berlin: Walter de Gruyter, 3. Aufl., 2003.

MATZKE (1922): Matzek, Josef. Die Landskroner Mundart. Landskron/Schönhengstgau: Josef Czerny, 1922.

MITZKA (1962): Mitzka, Walther. Schlesisches Wörterbuch. Berlin: Walter de Gruyter, 1962.

SCHWARZ (1958): Schwarz, Ernst. Probleme der sudetendeutschen Lehnwortgeographie, in: Zeitschrift für Mundartforschung, 1958, 26, S. 128-150.

WIESINGER, Peter (1980): "Sprache“, „Dialekt“ und „Mundart" als sachliches und terminologisches Problem, in: Dialekt und Dialektologie, 1980, S. 177-194.

ZEHETNER (1998): Zehetner, Ludwig. Bairisches Deutsch. München: Heinrich Hugendubel, 1998.

ZÜRRER(1999): Zürrer, Peter. Sprachinseldialekte. Aarau: Sauerländer Verlag, 1999. 